\title{
Postoperative Bilateral Palsy of Terminal Laryngeal and Pharyngeal Branches of the Vagal Nerve
}

\author{
Dirk Nauheimer $^{*}, 1$, Stephan Doering ${ }^{1}$, Thomas Breinlich ${ }^{2}$ and Goetz Geldner ${ }^{1}$ \\ ${ }^{I}$ Department of Anesthesiology and Intensive Care, Germany \\ ${ }^{2}$ Consultant Specialist in Otorhinolarngology, Germany
}

\begin{abstract}
We report a rare case of a postoperative bilateral laryngeal and pharyngeal lesion of the extracranial vagal nerve following a routine general anaesthesia in prone position. It documents a possible way of a serious complication depending to the positioning of the patient in prone position. Etiological factors and measures to avoid this rare but critical complication are discussed.

For the clinical practise it should give an advice to minimize the risk of injuries by malpositioning.
\end{abstract}

Keywords: Prone position, malpositioning, complications, vagal nerve lesion, general anaesthesia.

\section{CASE}

Perioperative complications such as nerve lesions secondary to malpositioning may lead to severe functional disability of the patient and reduce a successful surgical procedure. These regrettable injuries that result from stretching, retraction and/or compression to soft tissue and peripheral nerves are especially true for surgery in prone position $[1,2]$.

We present this rare case of a postoperative bilateral lesion of the extracranial vagal nerve following a routine general anaesthesia in prone position to demonstrate possible ways in which nerves can be damaged in the perioperative phase.

A 56 year old, $62 \mathrm{~kg}$ weighted woman with a 2 year history of frequent lower back pain presented increasing pain and hypaesthesia of both lower extremities as well as a dyfunction of the urinary bladder for the last 2 months. Radiological imaging showed the lumbal stenosis of L3/4 and L4/5 and the instable degenerative lumbal spondylodesis of L3-5. The neurological deficits indicated neurosurgical decompression of lumbal stenosis and transpedicular stabilization of the lumbal spondylodesis. At admission to the surgical treatment the patient was in good physiological condition. 4 years ago a chronic lymphatic leukaemia (CLL) was detected. The Bcell CLL was diagnosed in an early stage (smoldering Binet A) and was not treated with systemic chemotherapy. She presented a peripheral leucocyte count of 28,000 per $\mu 1$, 158,000 thrombocytes per $\mu \mathrm{l}$ and a hemoglobine count of $12,2 \mathrm{~g} / \mathrm{l}$. Different surgical procedures such as hysterectomy, and total endoprothesis of the right knee joint secondary to gonarthrosis were performed in the past years without significant complications.

We introduced general anaesthesia with $20 \mu \mathrm{g}$ of Sufentanil, $80 \mathrm{mg}$ of Propofol and $30 \mathrm{mg}$ of Rocuronium. The orotracheal intubation proved to be uncomplicated. The orotracheal

*Address correspondence to this author at the Department of Anesthesiology and Intensive Care, Hospital of Ludwigsburg, Posilipostr. 4, D-71640 Ludwigsburg, Germany; E-mail: nauhdi01@kliniken-lb.de tubus measured an internal diameter of $7,0 \mathrm{~mm}$ and was fixed on the left side of the mouth. The cuff was inflated with air up to $22 \mathrm{cmH}_{2} \mathrm{O}$ and cuff pressure was monitored continuously using a manometer connected to the cuff pilot balloon. Anaesthesia was maintained with Isoflurane $(0,6-$ 0,7 vol.\% in exspiration), air-oxygen-inflation with an oxygen-fraction of $100 \%$ before and $50 \%$ performed in low flow after positioning the patient as well as continuous perfusion of Remifentanil. Afterwards the patient was turned into prone position. The head was slightly tilted to the left side and placed on a special horseshoe pad to protect both eyes nose and neck. The upper extremities were placed neutral on soft pads. During the perioperative period respiratory and cardiopulmonary parameters, cuff pressure and core body temperature were continuously monitored. To keep the core temperature above $36^{\circ} \mathrm{C}$ and to prevent hypothermia the convective Warmtouch $^{\text {TM }}$ warming blankets were used. The duration of surgery was about 10,5 hours. The loss of blood was entirely about 5 litres and was substituted with 6 concentrates of erythrocytes, 1 litre of human plasma and 3,5 litres of hydroxyethyl starch. Neither vasopressors nor the application of katecholamines were needed to maintain the blood pressure (>100 $\mathrm{mmHg}$ systolic). At the end of surgery the patient was turned back from prone position and anaesthesia was discontinued. Under stable hemodynamic conditions (RR $>110 \mathrm{mmHg}$ systolic, $\mathrm{Hb} 10,9 \mathrm{~g} / \mathrm{dl}$ ) and adequate respiratory effort the tubus was removed. At the front of the lower neck, the upper thorax and the region above both superior spinae iliacae irritant cutaneous reactions were markable. Additionally pressure dependent blisters appeared at the right front of her neck. For observation she was transferred to the intensive care unit. Soon thereafter she developed an inspiratory marked stridor and dyspnea that was unresponsive to the intravenous application of $100 \mathrm{mg}$ solu decortin and nebulized epinephrine. Fibreoptic bronchoscopic examination confirmed swollen laryngeal mucous membranes, epiglottis and a submucosal hematoma of the vocal cords. About 3 hours after surgery she was awake reintubated with laryngobronchoscopic support. Anaesthesia was deepened again using continuous application of propo- 
fol. After extubation the following day the stridor was weakened but hoarseness and difficulty in swallowing still existed. Under forced respiratory effort the inspiratory stridor and dyspnea increased. Laryngoscopic examination revealed laryngeal stridor, paramedian positioning of the vocal cords with loss of function and loss of sensitivity of the supraglottic region.

The diagnosis of bilateral incomplete vagal nerve paralysis including laryngeal and pharyngeal vagal branches was made. Because of the increased risk of aspiration secondary to pharyngeal dysfunction a percutaneous endoscopic gastrostomy was made for nutrition. Hoarseness and difficulty in swallowing disappeared completely within about 100 days and gastrostomy was removed.

\section{DISCUSSION}

In this case we report a serious injury of the extracranial branches of the vagal nerve that appeared postoperatively after a long lasting surgery in prone position. Different factors such as the length of surgery, the positioning of the patient, cuff pressure and extension of the neck have been suggested as possible causes.

As in the presented case, nerve palsies may occur as a result of high pressure, compression, extension or trauma [1, 3].

The extent of this nerve palsy included the laryngeal branches as well as the pharyngeal branches of the vagal nerve.

Muscles and the mucous membrane of the larynx are innervated by the superior and recurrent laryngeal nerve. They originate from the vagal nerve. The superior laryngeal nerve divides into two branches. The external motor branch supplies the cricothyroid muscle. The internal sensory branch innervates the mucous membrane above the vocal cords. The recurrent laryngeal nerve loops on the right side under the subclavian artery and ascends between the trachea and esophagus to the larynx. On the left side, it loops under the arch of the aorta and ligamentum arteriosum and ascends in the tracheo-esophageal groove to the larynx. The nerve divides into a motor and sensory branch. It innervates all internal laryngeal muscles and the mucous membrane below the vocal cords $[4,5]$.

The pharyngeal branches of the vagal nerve include motor, sensory and secretory fibres that supply the oro- and laryngopharynx. They are part of the pharyngeal plexus that supplies fibres of the glossopharyngeal and the vagal nerve.

Several cases of bilateral vocal cord paralysis following endotracheal intubation or the use of laryngeal mask airways are reported and discussed [5,6]. Brandwein examined human cadaver larynges and found that the recurrent laryngeal nerve seemed to be vulnerable to compression between the arytenoid cartilage and the thyroid lamina. Compression secondary to cuff overinflation during anaesthesia using nitrous oxide may be a contributing factor. Increasing cuff pressure due to the diffusion of nitrous oxide may result in a stretching and direct pressure to the recurrent laryngeal and the external branch of the superior laryngeal nerve $[7,8]$. To ensure the appropriate cuff pressure and avoid overinflation a continuous monitoring should be used.
Furthermore the patient in this presented case had a chronic lymphatic leukaemia in an early stage. Smoldering CLL is characterized by normal hemoglobine and platelet values fewer than 2 areas of lymph node enlargement and peripheral blood lymphocytes of less than 30,000 per $\mu 1$. At that stage of the disease a therapeutic intervention does not prolong survival [9].

Rare but possible complications of lymphatic leukaemia are lesions of the peripheral or cranial nerves due to progressive malignancies or therapeutic interventions $[10,11]$. The bilateral vagal nerve palsy in the described case is in this context impropably caused by the smoldering B-cell CLL. Another aspect in the origin of nerve palsies is malpositioning. Complications associated with perioperative positioning of the patient are reported to occur in 1:1000 cases [12].

Specific modes of positioning the patient are essential for successful surgical procedures. Correct handling of patient positioning can minimize the risk of damage of nerves, vessels and soft tissue, although the possibility of injuries still exists. The ulnar nerve and the brachial plexus are at highest risk in positioning the extremities [13]. Especially the prone positioning requires great care and attention for head, neck and extremities. Injuries such as thoracic outlet syndrome caused by an unknown cervical rib, dislocation of the shoulder or the loss of vision because of an ischemic optic neuropathia are rare postoperative complications due to combination of factors and surgery in the prone position [14-16].

A combined bilateral lesion of the laryngeal and pharyngeal branches of the vagal nerve in association with a prone position has not been described in the literature before.

Major factors for the development of the described complication following spine surgery in prone position are malpositioning of head, neck and extremities and prolonged duration of surgery. Complications often also depend on preexisting non-symptomatic dysfunctions such as peripheral vascular disease or a cervical rib. To minimize the risk of damage by malpositioning, correct execution as well as constantly control of body position during surgery is necessary.

Prevention remains the main aspect of the management of positioning injuries.

In the presented case, several different factors seemed to be the reason for the complication.

The erythema of the skin at the anterior neck as well as pressure dependent blisters were remarkable and clearly showed that these regions were under increased external compression. Internal splinting through the cuffed tubus, as well as edema and hemtoma of the surrounding soft tissue secondary to the upper venous congestion, might be a further aspect. Dependent edema of head and neck can also be a consequence of prone position surgery. Turning the patient into prone position increases the compression on to the thorax. Increased intrathoracic pressure can lead to increased central venous pressure, decreased venous return and decreased cardiac output [17]. In addition to other aspects the duration of the surgery seemed to be important. Although head and neck were positioned correctly at the beginning of the procedure and positioning controls were performed all 30 minutes of the surgery this paralysis was not obvious while employing the prone position. 
To prevent such critical complications due to mal positioning first attention should be focused on a safe transition from supine into the prone position, that includes head and neck positioning, eye, ear and nose protection, the monitoring of the endotracheal tube and the anatomical placement of upper and lower extremities. Special horseshoe pads for placement of the head keeps pressure off eyes and ears and allows mobility and adjusting of the neck. Be aware of severe rotation and overextension of the neck. The head should be placed above the level of the heart to prevent increased venous stasis especially for prolonged surgery. The upper and lower extremities have to be ensured and padded in neutral position.

Nevertheless it is necessary to control and document the positioning of the patient to avoid complications and ensuing healthy and forensic consequences [18].

\section{REFERENCES}

[1] Ullrich W, Biermann E, Kienzle F, Krier C. [Damage due to patient positioning in anesthesia and surgical medicine (1)]. Anasthesiol Intensivmed Notfallmed Schmerzther 1997; 32 (1): 4-20.

[2] Anderton JM. The prone position for the surgical patient: a historical review of the principles and hazards. Br J Anaesth 1991; 67 (4): 452-63.

[3] Winfree CJ, Kline DG. Intraoperative positioning nerve injuries. Surg Neurol 2005; 63 (1): 5-18; discussion 18.

[4] Robinson PM, Weir AM. Interobserver variability in assessment of the larynx. Clin Otolaryngol Allied Sci 1987; 12 (6): 413-5.

[5] Zealear DL, Billante CR. Neurophysiology of vocal fold paralysis. Otolaryngol Clin North Am 2004; 37 (1):1-23.
[6] Brandwein M, Abramson AL, Shikowitz MJ. Bilateral vocal cord paralysis following endotracheal intubation. Arch Otolaryngol Head Neck Surg 1986; 112 (8): 877-82.

[7] Sacks MD, Marsh D. Bilateral recurrent laryngeal nerve neuropraxia following laryngeal mask insertion: a rare cause of serious upper airway morbidity. Paediatr Anaesth 2000; 10 (4): 435-7.

[8] Daya H, Fawcett WJ, Weir N. Vocal fold palsy after use of the laryngeal mask airway. J Laryngol Otol 1996; 110 (4): 383-4.

[9] Dighiero G, Hamblin TJ. Chronic lymphocytic leukaemia. Lancet 2008; 371 (9617): 1017-29.

[10] Shibata-Hamaguchi A, Samuraki M, Furui E, et al. B-cell neurolymphomatosis confined to the peripheral nervous system. J Neurol Sci 2007; 260 (1-2): 249-52.

[11] Buyukavci M, Tan H, Akdag R. An alarming sign for serious diseases in children: bilateral facial paralysis. Pediatr Neurol 2002; 27 (4): 312-3.

[12] Bund M, Heine J, Jaeger K. [Complications due to patient positioning: anaesthesiological considerations]. Anasthesiol Intensivmed Notfallmed Schmerzther 2005; 40 (6): 329-39.

[13] Winfree CJ. Peripheral nerve injury evaluation and management. Curr Surg 2005; 62 (5): 469-76.

[14] Ali AA, Breslin DS, Hardman HD, Martin G. Unusual presentation and complication of the prone position for spinal surgery. J Clin Anesth 2003; 15(6): 471-3.

[15] Corcia P, Guennoc AM, Barthez MA, de Courtivon B, de Toffol B, Laulan J. [Thoracic outlet syndrome: an unusual postoperative complication]. Rev Neurol (Paris) 2006; 162 (2): 240-2.

[16] Kamming, D, Clarke S. Postoperative visual loss following prone spinal surgery. Br J Anaesth 2005; 95 (2): 257-60.

[17] Mobley SR, Miller BT, Astor FC, Fine B, Halliday NJ. Prone positioning for head and neck reconstructive surgery. Head Neck 2007; 29 (11): 1041-5.

[18] Weissauer W. [Allocation of responsibility for positioning patients for surgery and liability for damages consequent on faulty positioning]. Anaesthesist 2002; 51 (3): 166-74. 